\section{The zebrafish organizer requires chordino}

The dorsoventral pattern of vertebrate embryos is established and regulated by opposing gradients of ventralizing bone morphogenetic proteins (BMPs) and BMP antagonists $^{1-3}$ (such as Chordin ${ }^{2,4}$, Noggin ${ }^{5,6}$ and Follistatin ${ }^{7,8}$ in the frog embryo) secreted by a dorsal organizer. In the zebrafish, Danio rerio, mutations in a number of genes that affect dorsoventral patterning of the early embryo have been identified $^{9,10}$, but only two, dino and mercedes, are involved in dorsal specification ${ }^{11}$, with dino mutants displaying the stronger, ventralized phenotype. Here we show that

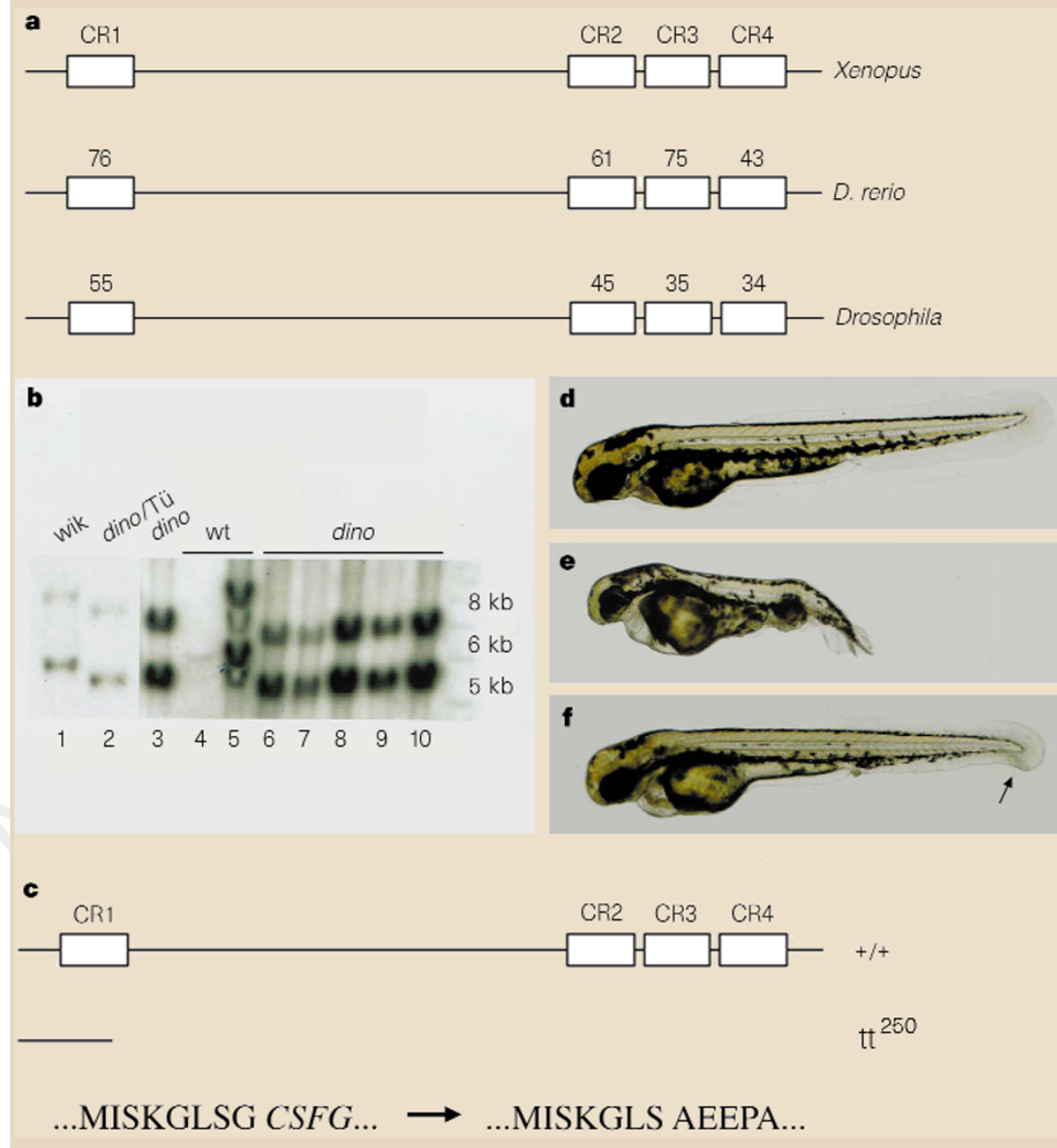

Figure 1 a, Schematic representation of Xenopus Chordin, zebrafish Chordin, and Drosophila Sog. Numbers indicate the percentage of amino-acid identity between the respective cysteine-rich domains. b, Linkage analysis, carried out as in ref. 15. Genomic DNA was digested with EcoRl. Lanes 1 and 2: parental DNA from the WK strain and a heterozygous dino individual in the Tübingen background, respectively. Lanes 3, 6-10: DNA from pools of dino mutant $F_{2}$ embryos (a total of 136 genome equivalents; 272 mutant chromosomes). Lanes 4 and 5: DNA from pools of sibling $F_{2}$ embryos $(+/+$ and din $/+; 0.5$ and 24 genome equivalents, respectively). c, Predicted structure of $\operatorname{din}^{\text {t250 }}$, containing the first 42 wild-type amino acids and 54 additional unrelated residues. Part of the amino-acid sequence of the wild type (left) and mutant protein (right) is shown, with the first four amino acids of CR1 displayed in italics. d, Wild-type embryo at 3 days of development. e, Sibling dino mutant embryo. Note the small eyes and split ventral tail fin. f, Mutant embryo, rescued by injection of Xenopus chordin mRNA. The ventral tail fin is partially duplicated (arrow), but otherwise the embryo appears normal.
In a restriction-fragment length polymorphism (RFLP) linkage analysis of more than 400 mutant chromosomes, we saw no recombination between dino and chordin (Fig. 1b), indicating that dino and chordin are within 0.25 centimorgans $(\mathrm{cM})$. The chordin complementary DNA from din ${ }^{t t 250}$ mutant embryos has a deletion of 104 base pairs, causing a frame-shift predicted to produce a severely truncated protein (Fig. 1c). This truncated form has only the first 42 amino acids of the wild-type Chordin protein and completely lacks the cysteinerich repeats.

Injection of Xenopus chordin messenger RNA into dino mutants rescues the mutant phenotype, producing viable and fertile homozygous dino mutant adult fish (Fig. 1 $\mathrm{d}, \mathrm{e}, \mathrm{f})$. This indicates that chordin is not required after the early stages of development, as the injected RNA is largely degraded by the end of the gastrulation stage (data not shown). These data indicate that the defects of dino mutant embryos are potentially caused by a null mutation in the chordin gene. In view of this genetic relationship, we will refer to both the mutant and the gene as chordino.

In mutant embryos, chordino expression is strongly reduced at midgastrula stages (Fig. 2a,b), indicating that chordino expression requires functional Chordino protein. Indeed, the wild-type chordino expression pattern can be rescued in chordino mutants by the injection of both Xenopus chordin mRNA (Fig. 2c) and mRNA encoding a dominant negative BMP receptor (Fig. 2d), whereas over-expression of $b m p-4$ in wildtype embryos leads to a reduction in chordino expression levels similar to those in chordino mutants (data not shown). This suggests that such autoregulation of chordino is achieved by an inhibition of antago-

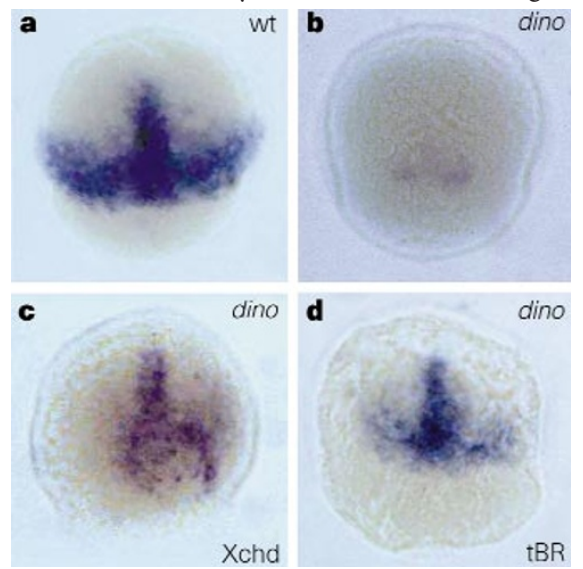

Figure 2 a, Wild-type and b, Mutant embryo. chordino mRNA can be rescued to almost wild-type levels by the injection of mRNA encoding $\mathbf{c}$, Xenopus chordin (Xchd) or $\mathbf{d}, \mathrm{A}$ dominant-negative BMP receptor (Br). All embryos are shown in a dorsal view after in situ hybridizations with a chordino antisense probe. a,b,d, $70 \%$ epiboly. c, $80 \%$ epiboly. RNA injections were done as in ref. 3 . 
nizing BMP signals which normally act as negative regulators of chordino expression.

In addition, chordino mutants display a dorsally expanded $b m p-4$ expression domain $^{3}$, indicating that during normal development chordino has a negative influence on $b m p-4$ transcription in dorsolateral regions. Again, this negative regulation might be mediated by BMPs themselves, which normally act as positive regulators of their own expression ${ }^{3}$.

It has been shown that Xenopus Chordin inhibits the antagonizing Bmp-4 activity by direct binding to the Bmp-4 protein, thereby preventing receptor activation ${ }^{4}$. Our data demonstrate that this inhibition at the protein level is potentiated by two synergistic feedback loops which lead to a derepression of chordino transcription and an inactivation of bmp-4 expression.

Our findings demonstrate that chordino is required during early dorsoventral patterning of the zebrafish embryo, where it functions as an antagonist of ventralizing BMP signals. This supports the notion that mechanisms of early dorsoventral patterning are conserved between vertebrates and invertebrates ${ }^{13}$.

\section{Stefan Schulte-Merker}

MPI für Entwicklungsbiologie, Spemannstrasse 35,

72076 Tübingen, Germany

\section{Kevin J. Lee}

Howard Hughes Medical Institute,

Department of Biochemistry and Molecular Biophysics,

Center for Neurobiology and Behavior, Columbia University, New York,

New York 10032, USA

Andrew P. McMahon

Department of Molecular and Cellular Biology,

Harvard University, 16 Divinity Avenue,

Cambridge, Massachusetts 02138, USA

Matthias Hammerschmidt ${ }^{*}$

MPI für Immunbiologie, Stübeweg 51,

79108 Freiburg, Germany

e-mail: hammerschmid@immunbio.mpg.de

* To whom correspondence should be addressed.

1. Graff, J. M., Thies, R. S., Song, J. J., Celeste, A. J. \& Melton, D. A. Cell 79, 169-179 (1994).

2. Sasai, Y., Lu, B., Steinbeisser, H. \& De Robertis, E. M. Nature 376, 333-336 (1995).

3. Hammerschmidt, M., Serbedzija, G. N. \& McMahon, A. P. Genes Dev. 10, 2452-2461 (1996)

4. Piccolo, S. Y., Sasai, Y., Lu, B. \& De Robertis, E. M. Cell 85, 589-598 (1996)

5. Re'em-Kalma, Y., Lamb, T. \& Frank, D. Proc. Natl Acad. Sci. USA 92, 12141-12145 (1995).

6. Zimmerman, L. B. \& Harland, R. M. Cell 85, 599-606 (1996).

7. Hemmati-Brivanlou, A., Kelly, O. G. \& Melton, D. A. Cell 77, 283-295 (1994)

8. Fainsod, A. et al. Mech. Dev. (in the press).

9. Haffter, P. et al. Development 123, 1-36 (1996).

10. Driever, W. et al. Development 123, 37-46 (1996).

11. Hammerschmidt, M. et al. Development 123, 95-102 (1996).

12. Francois, V., Solloway, M., O’Neill, J. W., Emery, J. \& Bier, E. Genes Dev. 8, 2602-2616 (1994)

13. Holley, S. A. et al. Nature 376, 249-253 (1995).

14. Biehs, B., Francois, V. \& Bier, E. Genes Dev. 10, 2922-2934 (1996).

15. Schulte-Merker, S., Eeden, F. V., Halpern, M. E., Kimmel, C. B. \& Nüsslein-Volhard, C. Development 120, 1009-1015 (1994).

\section{Have quantum scars been observed?}

Wilkinson et al. ${ }^{1}$ have reported observations of oscillations in the current of a resonant tunnelling diode in a magnetic field. They proposed that this provides the first direct experimental evidence for quantum 'scarring' in a real chaotic system. But we believe that Wilkinson et al. have not observed quantum scars in the usual sense. They considered a far-from semiclassical regime where current theories of scarring are not valid. Demonstrating these effects in a solidstate device is a tremendous achievement. However, we believe that even in the semiclassical limit this type of experiment does not go beyond other experiments on atoms in magnetic fields as a probe of scarring.

A quantum particle travelling from A to $\mathrm{B}$ experiences all possible paths in between. The wave-like nature of a quantum particle means that each path has a phase and so neighbouring paths interfere with each other. The combined contributions of these paths build up the wavefunction, which provides a complete description of the quantum the semiclassical limit $(\hbar \rightarrow 0)$, phases associated with different paths are large. Hence, neighbouring paths can rapidly fall out of phase and their contributions cancel by destructive interference. When the typical motion is a chaotic trajectory, this should produce wavefunctions with no structure other than a random grainy pattern.

But Heller ${ }^{2}$ found that for $\hbar \rightarrow 0$, quantum states of a chaotic system show 'scars', concentrations near the paths of unstable, isolated periodic orbits (classical orbits which retrace themselves and are isolated if there is no other periodic orbit of similar phase nearby). The issue of how and when quantum states are scarred in the semi-

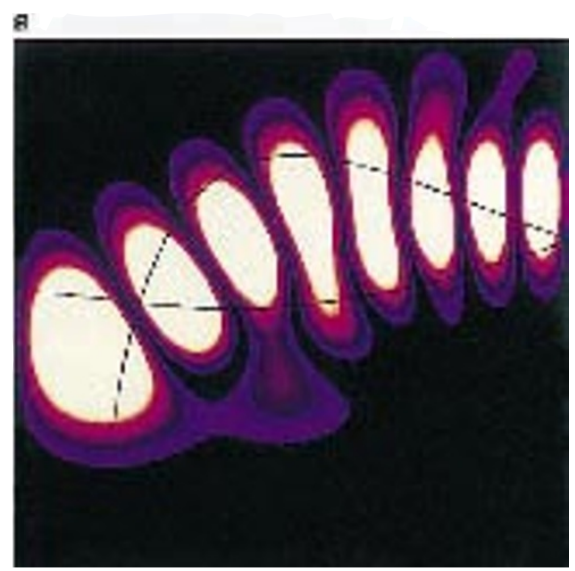
dynamics of the particle. At high energies, in

classical limit has sparked a lively debate. The mathematical theories all assume that the periodic orbits are isolated, though more than one isolated orbit can scar a single state.

Wilkinson et al. have probed the 25 or so lowest quantum levels above the ground state. By lowering the energy, the phases of the classical orbits are made small, so that they remain in-phase. Hence, the neighbourhoods of topologically distinct periodic orbits become so large that they overlap and so cease to be isolated.

In the experiment, the electronic motion is confined by a magnetic field to a cylindrical energy surface whose ends are the two barriers of the well. Wilkinson et al. found that the tunnelling is dominated by four single eigenstates, roughly 'linear' in shape with $N \approx 10$ oscillations along the magnetic field. However, the energy surface supported only $n \approx 2-3$ oscillations perpendicular to the field, so each 'linear' eigenstate is supported by a large fraction of the Poincaré surface on the emitter wall, including a cluster of short periodic orbits of similar action that traverse the length of the cylinder.

This similarity of action is analogous to back and forth motion close to the axis of a long, thin cylinder. Most of the action comes from the longitudinal motion, with smaller differences due to the transverse components. The periodic orbits become isolated only when we can resolve the differences in action (typically for $n>10$ ). For small $n$ (non-isolated case), sequences of individual 'linear' states, roughly aligned with the magnetic field, are ubiquitous ${ }^{1,3}$. These states cannot distinguish between the different periodic orbits. The stability parameters of the classical dynamics are essentially irrelevant, in contrast to an isolated scar. They are essentially a quantum phenomenon, the socalled adiabatic separability ${ }^{4}$, more relevant in the deep quantum regime than an interpretation in terms of scars. A linear state $(n \approx 1)$ is shown in Fig. 1a, and Fig. 1b shows a scar in the semiclassical regime $(n \approx 30)$.

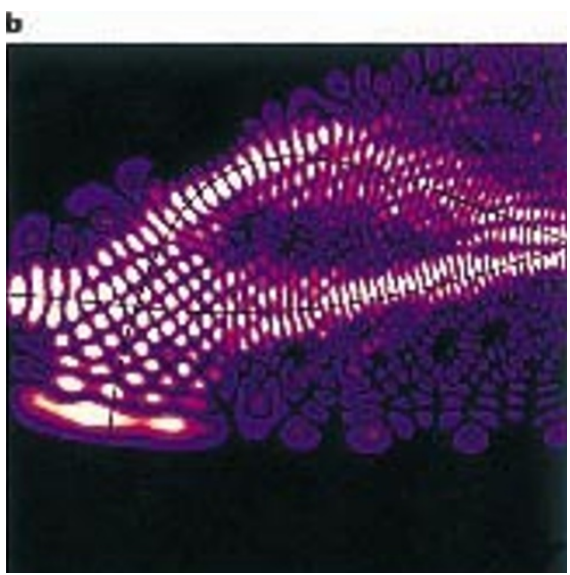

Figure 1 Quantum eigenstates which dominate tunnelling for: $\mathbf{a}, n \approx 1$ far from the semiclassical limit showing 'linear' quantization; and $\mathbf{b}, n \approx 30$ showing scarring by the $S_{1}$ unstable periodic orbit observed in widewell experiments. We ensured that both states corresponded to exactly the same set of classical periodic orbits, differing only in effective size of $\hbar$ by using a scaling property of the dynamics. 